\title{
Thermophysical measurements on transition- metal tungstates \\ II. Heat capacities of antiferromagnetic nickel and cobalt tungstates ${ }^{b}$
}

\author{
CHRISTOPHER P. LANDEE and EDGAR F. WESTRUM, JR. ${ }^{\mathfrak{c}}$ \\ Department of Chemistry, The University of Michigan, Ann Arbor, \\ Michigan 48104, U.S.A.
}

(Received 3 November 1975; in revised form 30 December 1975)

\begin{abstract}
The heat capacity of nickel tungstate $\left(\mathrm{NiWO}_{4}\right)$ was measured over the temperature range 5 to $350 \mathrm{~K}$ by adiabatic calorimetry, and that of cobalt tungstate $\left(\mathrm{CoWO}_{4}\right)$ from 5 to $550 \mathrm{~K}$. Temperatures of maximum heat capacities for the antiferromagnetic anomalies in cobalt and nickel tungstates were $(47.7 \pm 0.1) \mathrm{K}$ and $(59.80 \pm 0.05) \mathrm{K}$. Excess entropies associated with the antiferromagnetic anomalies were evaluated as $R \ln 2\left(1.38 \mathrm{cal}_{\mathrm{cn}} \mathrm{K}^{-1} \mathrm{~mol}^{-1}\right)$ for CoWO and $R \ln 3\left(2.18 \mathrm{cal}_{\mathrm{th}} \mathrm{K}^{-1} \mathrm{~mol}^{-1}\right)$ for $\mathrm{NiWO}_{4}$ with the lattice heat capacities of the compounds approximated by that of $\mathrm{ZnWO}_{4}$. In addition, the heat capacity of $\mathrm{CoWO}_{4}$ showed a continuing excess heat capacity consistent with a Schottky anomaly from energy levels lying roughly 600 to $3400 \mathrm{cal}_{\mathrm{tn} \mathrm{mol}}^{-1}$ above the ground state. Selected thermal functions: $C_{\triangleright}^{\circ}, S^{\circ}$, and $-\left\{G^{\circ}(T)-H^{\circ}(0)\right\} / T$ at $298.15 \mathrm{~K}$ are respectively $28.41,30.08$, and 14.01 $\mathrm{cal}_{\mathrm{th}} \mathrm{K}^{-1} \mathrm{~mol}^{-1}$ for CoWO , and 27.77, 28.51, and $13.21 \mathrm{cal}_{\mathrm{tn}} \mathrm{K}^{-1} \mathrm{~mol}^{-1}$ for $\mathrm{NiWO}_{4}$.
\end{abstract}

\section{Introduction}

The first-row transition-element metal tungstates: $\mathrm{MnWO}_{4}, \mathrm{FeWO}_{4}, \mathrm{CoWO}_{4}$, $\mathrm{NiWO}_{4}$, and $\mathrm{ZnWO}_{4}$, form an isostructural series of compounds in which the number of $3 \mathrm{~d}$ electrons increases from 5 in $\mathrm{MnWO}_{4}$ to 10 in $\mathrm{ZnWO}_{4}$. The crystal structure of these compounds is of the $\mathrm{NiWO}_{4}$-type, ${ }^{(1)}$ space group $\mathrm{P} 2 / \mathrm{c}\left(\mathrm{C}_{2 \mathrm{~h}}^{4}\right)$ and the lattice parameters increase gradually (but irregularly) along the series. ${ }^{(2)}$ Because the 3d shell is incomplete, $\mathrm{MnWO}_{4}, \mathrm{FeWO}_{4}, \mathrm{CoWO}_{4}$, and $\mathrm{NiWO}_{4}$ are paramagnetic at $300 \mathrm{~K}$. At low temperatures, the compounds undergo cooperative transitions to antiferromagnetically-ordered states as shown by both magnetic susceptibility measurements ${ }^{(3-5)}$ and magnetic structures determined by neutron diffraction. ${ }^{(6-8)}$ $\mathrm{FeWO}_{4}, \mathrm{CoWO}_{4}$, and $\mathrm{NiWO}_{4}$ share a common magnetic structure in which the moments lie in the $a c$-plane, inclined to the $a$-axis by angles of $29^{\circ}$ for $\mathrm{FeWO}_{4},{ }^{(7)}$ between 20 and $45^{\circ}$ for $\mathrm{CoWO}_{4},{ }^{(8-10)}$ and $15^{\circ}$ for $\mathrm{NiWO}_{4} .^{(11)}$ The magnetic cell is double the crystallographic unit cell along the $a$-axis with the two moments in each

a The previous paper in the series is reference 14

- This work has been supported by the National Science Foundation, contracts NSF GP-33424X. and NSF GP-42525X.

- To whom correspondence concerning this paper should be addressed. 
cell parallel to one another and antiparallel to the pairs adjacent to them along the $a$-axis. This arrangement implies ferromagnetic layers within the chains in each $b c$-plane, coupled antiferromagnetically to the adjacent planes (compare figure 1). The magnetic space group (Shubnikov group) for this structure is $P_{c} 2 / c$. The more complicated magnetic structure of $\mathrm{MnWO}_{4}$ is discussed by Dachs. ${ }^{(12)}$

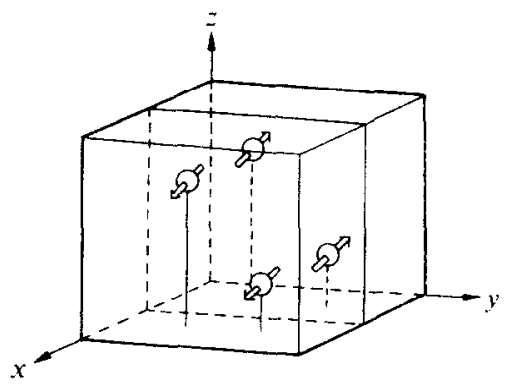

FIGURE 1. Magnetic structure of iron (II), nickel, and cobalt tungstates, after Ưlkü. ${ }^{(7)}$

Measurement of the heat capacities of magnetic compounds yields valuable information concerning the magnetic disordering process as well as the magnetic entropy. The observed heat capacity is a combination of the magnetic disordering, of lattice vibrations, and-sometimes-of the population of nearby excited electronic states. To estimate the lattice contribution to the total heat capacity, the heat capacity of the isomorphous diamagnetic compound, $\mathrm{ZnWO}_{4}$, is used. ${ }^{(13,14)}$

\section{Experimental}

\section{SAMPLE PREPARATION AND CHARACTERIZATION}

Cobalt tungstate. The sample of cobalt tungstate purchased from Alfa Inorganics who had obtained it from Sylvania, Inc., was labelled "Crystal Grade", and had a stated purity of 99.96 moles per cent. The material was amorphous and showed no diffraction lines in preliminary X-rays analyses, but after firing in air at $1120 \mathrm{~K}$ for $20 \mathrm{~h}$ in a platinum crucible yielded a sharp X-ray pattern which revealed the presence of $\mathrm{WO}_{3}$ impurity lines in addition to the $\mathrm{CoWO}_{4}$ lines. Since $\mathrm{WO}_{3}$ is soluble in alkali, the $\mathrm{CoWO}_{4}$ was rinsed in $1 \mathrm{M} \mathrm{KOH}(\mathrm{aq})$, distilled water, then in $1 \mathrm{M} \mathrm{HCl}(\mathrm{aq})$ and again in water. $\dagger$ After drying in a drying oven, the sample was pressed to a pellet with $120 \mathrm{MPa}$ pressure and fired for $30 \mathrm{~h}$ at $1275 \mathrm{~K}$.

Since X-ray-diffraction analysis showed faint traces of $\mathrm{WO}_{3}$ still present in the sample, the CoWO $\mathrm{CW}_{4}$ was leached in $50 \mathrm{~cm}^{3}$ batches in $400 \mathrm{~cm}^{3}$ of $1 \mathrm{M} \mathrm{KOH(aq)}$ for $15 \mathrm{~min}$. Each batch was then filtered, rinsed in distilled water, rinsed in $0.2 \mathrm{M}$ $\mathrm{HCl}(\mathrm{aq})$ (to remove traces of alkali), and finally rinsed in distilled water. The sample was pelletted and fired as before. Subsequent $\mathrm{X}$-ray-diffraction analyses showed no impurity lines and the lattice parameters derived for the $\mathrm{CoWO}_{4}$ are in good accord

† Throughout this paper $M=\mathrm{mol} \mathrm{dm}^{-3} ; \mathrm{cal}_{\mathrm{th}}=4.184 \mathrm{~J}$; Torr $=(101.325 / 760) \mathrm{kPa}$. 
HEAT CAPACITIES OF NICKEL AND COBALT TUNGSTATES

TABLE 1. Derived lattice parameters of cobalt tungstate

\begin{tabular}{|c|c|c|c|c|}
\hline$a / \mathrm{nm}$ & $b / \mathrm{nm}$ & $c / n m$ & $\beta$ & Reference \\
\hline $\begin{array}{l}0.467 \pm 0.001 \\
0.4669 \pm 0.0003 \\
0.4667 \pm 0.0001 \\
0.4666 \pm 0.0002\end{array}$ & $\begin{array}{l}0.569 \pm 0.001 \\
0.5683 \pm 0.0003 \\
0.5681 \pm 0.0001 \\
0.5680 \pm 0.0002\end{array}$ & $\begin{array}{l}0.494 \pm 0.001 \\
0.4948 \pm 0.0003 \\
0.4947 \pm 0.0001 \\
0.4948 \pm 0.0002\end{array}$ & $\begin{array}{l}90^{\circ} \\
90^{\circ} \\
90^{\circ} \\
90^{\circ}\end{array}$ & $\begin{array}{l}\text { Broch(15) }^{(15)} \\
\text { Swanson ef al. } \\
\text { Sleight }^{(2)} \\
\text { This research } \\
\text { (Guinier, Cu } \mathrm{K} \alpha \text { ) }\end{array}$ \\
\hline
\end{tabular}

with those of other investigators also given in table 1. Further details of the X-ray pattern obtained for both $\mathrm{CoWO}_{4}$ and $\mathrm{NiWO}_{4}$ are available elsewhere. ${ }^{(17)}$

The results of professional chemical analysis for cobalt and tungsten are listed in table 2 for two separate batches. Both the cobalt and tungsten are reported to be low with mole ratio $n(\mathrm{Co}) / n(\mathrm{~W})$ of $(0.978 \pm 0.020)$. If $\mathrm{WO}_{3}$ were the only impurity present, the mole ratio would be low but the mass percentage of tungsten would exceed the theoretical value for cobalt tungstate, rather than be only 98.8 per cent

TABLE 2. Composition of transition metal tungstates, $\mathrm{MWO}_{4}$ : mass fraction $f$ and amount of substance $n$

\begin{tabular}{|c|c|c|c|}
\hline $\begin{array}{l}\text { Compound } \\
\text { and sample }\end{array}$ & $10^{2} f(\mathrm{M})^{a}$ & $10^{2} f(W)^{\circ}$ & $n(\mathrm{M}) / n(\mathrm{~W})$ \\
\hline &  & $-\cdots-$ & $--\cdots \cdots$ \\
\hline $\begin{array}{l}\mathrm{CoWO}_{4}-\mathrm{II}^{\prime} \\
\text { CoWO }_{4}-\mathrm{III}^{\prime} \\
\text { Theoretical }\end{array}$ & $\begin{array}{l}18.7 \pm 0.03 \\
18.6 \pm 0.3 \\
19.21\end{array}$ & $\begin{array}{l}59.2 \pm 0.3 \\
59.2 \pm 0.3 \\
59.90\end{array}$ & $\begin{array}{l}0.986 \pm 0.02 \\
0.978 \pm 0.02 \\
1.000\end{array}$ \\
\hline $\begin{array}{l}\mathrm{NiWO}_{4} \\
\text { Theoretical }\end{array}$ & $\begin{array}{l}21.3 \pm 0.3^{d} \\
19.15\end{array}$ & $\begin{array}{l}58.3 \pm 0.3^{d} \\
59.97\end{array}$ & $\begin{array}{l}1.14 \pm 0.02 \\
1.000\end{array}$ \\
\hline
\end{tabular}

a Cationic metal by titration with EDTA.

- Spectrophometric determination for tungsten as tetrathiocyanato tungsten by the method of Gottschalk, $Z$. Anal. Chem. 1962, 187, 164.

- Calorimetric sample.

¿ Average of three determinations.

of theoretical. A composition of the form $\left(\mathrm{CoWO}_{4}+x \mathrm{WO}_{3}+\gamma\right)$, where $\gamma$ contains neither cobalt nor tungsten, would require 1.6 mass per cent of $\mathrm{WO}_{3}$ and 1.8 mass per cent of $\gamma$ to duplicate the analytical results. The sensitivity of the X-ray analysis used is sufficient to rule out such a possibility, so the analytical results must be considered questionable.

To test for the possible presence of volatile impurities (water, etc.) a sample of several $\mathrm{mg}$ was heated to $1475 \mathrm{~K}$ under a nitrogen atmosphere in a Perkin-Elmer TGS-1 Thermobalance. No mass loss $( \pm 0.08)$ per cent was observed.

The presence of other metal impurities was tested in both batches II' $^{\prime}$ and III' $^{\prime}$ with a Perkin-Elmer 503 Atomic Absorption Spectrophotometer. Trace metals determined (mass fractions: $74 \times 10^{-6}$ of cobalt, $524 \times 10^{-6}$ of iron, $102 \times 10^{-6}$ of 
nickel, and $77 \times 10^{-6}$ of zinc) yield an accumulated mass fraction of impurity less than 0.08 per cent so that compounds of these metals do not explain the discrepancy in the bulk analyses.

Atomic absorption spectroscopy was used to determine the mass percentage of cobalt in the sample. The reliability of this determination was low from background noise level within the instrument and problems of preparing consistent standards. Yet the analysis for cobalt gives $(98.7 \pm 0.8)$ mass per cent of the theoretical value. This value, when used with the commercially determined mass percentage of tungsten, gives $n(\mathrm{Co}) / n(\mathrm{~W})=(1.00 \pm 0.01)$, close to unity.

Nickel tungstate. The sample of "Crystal Grade" nickel tungstate purchased from Alfa Inorganics had been manufactured by Sylvania, Inc. and had a reported purity of 99.96 moles per cent. When the sample was first investigated, it was a light-green amorphous powder. After annealing for $24 \mathrm{~h}$ at $1125 \mathrm{~K}$ the color changed to peagreen and X-ray exposure for $2 \mathrm{~h}$ revealed a pattern of sharp lines. Most lines could be indexed as $\mathrm{NiWO}_{4}$ reflexions but three lines corresponded to reflexions from NiO. ${ }^{(18)}$ A later exposure for $8 \mathrm{~h}$ on more sensitive Ilford Type-G film revealed the presence of additional impurity lines in the original material corresponding to reflexions from $\mathrm{CaWO}_{4},{ }^{(19)}$ and from $\mathrm{Na}_{2} \mathrm{WO}_{4} \cdot{ }^{(20)}$ An additional $\mathrm{NiO}$ line appeared. However, the presence of the calcium tungstate and sodium tungstate were not known during the purification undertaken to remove the nickel oxide impurity.

The calorimetric sample of $\mathrm{NiWO}_{4}$ was prepared in the following manner. The original material was pressed into a pellet, annealed overnight in a tube furnace at $1050 \mathrm{~K}$, and then crushed in an agate mortar. The powder was poured into a ball mill, distilled water added to make a slurry, and the sample milled for $2 \mathrm{~d}$ to crush the powder. After removal from the mill and drying, the sample was forced through a $0.25 \mathrm{~mm}$ sieve, boiled for $15 \mathrm{~min}$ in $1 \mathrm{M} \mathrm{HCl}(\mathrm{aq})$ to leach out $\mathrm{NiO}$. This acid wash changes the color of the sample from dark green to mustard yellow. The material was filtered, rinsed in distilled water, dried, and then given a final annealing at $1170 \mathrm{~K}$ for $14 \mathrm{~h}$ in a Vycor tube. The color of the sample changed to a dark gold during this annealing. A preliminary $\mathrm{X}$-ray at this point showed the absence of all $\mathrm{NiO}$ lines, but very faint $\mathrm{WO}_{3}$ lines persisted. Consequently, the material was boiled in $1 \mathrm{M} \mathrm{KOH}(\mathrm{aq})$, filtered, rinsed in distilled water, and dried.

Subsequently, X-ray studies yielded lattice parameters generated from the refined data shown in table 3 which agreed well with those of other investigators. Long-

TABLE 3. Derived lattice parameters of nickel tungstate

\begin{tabular}{|c|c|c|c|c|}
\hline$a / \mathrm{nm}$ & $b / \mathrm{nm}$ & $c / n m$ & $\beta$ & Reference \\
\hline $\begin{array}{l}0.469 \pm 0.001 \\
0.460 \pm 0.0015 \\
0.4600 \pm 0.0004 \\
0.4599 \pm 0.0001 \\
0.4600 \pm 0.0002\end{array}$ & $\begin{array}{l}0.567 \pm 0.001 \\
0.566 \pm 0.002 \\
0.5665 \pm 0.0004 \\
0.5665 \pm 0.0001 \\
0.5663 \pm 0.0002\end{array}$ & $\begin{array}{l}0.494 \pm 0.001 \\
0.491 \pm 0.002 \\
0.4912 \pm 0.0004 \\
0.4910 \pm 0.0001 \\
0.4911 \pm 0.0002\end{array}$ & $\begin{array}{l}90.33^{\circ} \\
(90.08 \pm 0.08)^{\circ} \\
90^{\circ} \\
90^{\circ} \\
90^{\circ}\end{array}$ & $\begin{array}{l}\text { Broch }^{(15)} \\
\text { Keeling(1) }^{(1)} \\
\text { Swanson et al. } \\
\text { Sleight }^{(2)} \\
\text { This research } \\
\text { (Guinier, Cu K } \alpha \text { ) }\end{array}$ \\
\hline
\end{tabular}


exposure irradiations revealed three impurity lines but no $\mathrm{NiO}$ and $\mathrm{WO}_{3}$ reflexions. Two of these are matched by lines of the $\alpha$-cristobalite phase of $\mathrm{SiO}_{2}$ which entered the sample during the final annealing in the Vycor tube. Where the sample had been in contact with the tube, a hard opaque coating formed which, when scraped from the inside of the tube and X-rayed, was observed to be pure $\alpha$-cristobalite, evidently produced by mineralizing action of $\mathrm{NiWO}_{4}$ on (amorphous) Vycor. The other impurity line is attributed to the persistent presence of calcium tungstate. The diminished relative abundance of the $\mathrm{CaWO}_{4}$ in the final sample, as determined by the number and intensity of the X-ray reflexions observed, is attributed to the slight solubility in water of this contaminant and the frequent rinses in distilled water the sample received. The absence of sodium tungstate reflexions is consistent with its higher solubility in water.

The nickel tungstate sample was chemically analyzed for the presence of silicon, calcium, and sodium. The assumption that these contaminants are present as the following compounds, implies impurity levels of 0.56 mass per cent $(2.80$ moles per cent) of $\mathrm{SiO}_{2}, 0.20$ mass per cent $\left(0.21\right.$ mole per cent) of $\mathrm{CaWO}_{4}$, and 0.08 mass per cent ( 0.08 mole per cent) of $\mathrm{Na}_{2} \mathrm{WO}_{4}$.

The results of chemical analysis for nickel and tungsten are listed in table 2. The value for nickel is high and that for tungsten is low; the calculated mole ratio $n(\mathrm{Ni}) / n(\mathrm{~W})$ is $(1.14 \pm 0.02)$. Since the $\mathrm{X}$-ray-determined lattice parameters are in such good accord with the previously published values, and since the sensitivity of the X-ray technique is sufficiently great to determine the 0.2 mole per cent of $\mathrm{CaWO}_{4}$ impurity, this anomalous $n(\mathrm{Ni}) / n(\mathrm{~W})$ mole ratio is not understood.

\section{HEAT-CAPACITY MEASUREMENTS}

Heat-capacity measurements in the range 5 to $350 \mathrm{~K}$ were made in the Mark II adiabatic cryostat ${ }^{(21)}$ while those made in the 300 to $500 \mathrm{~K}$ range were made in the Mark IV adiabatic thermostat. ${ }^{(2)}$ Relevant loading information for the samples is given in table 4 .

TABLE 4. Calorimeter loading information:

calorimeter volume $V$, sample mass $m$, pressure $p$ of helium gas, and density $\rho$ of the sample

[Torr $=(101.325 / 760) \mathrm{kPa}]$

\begin{tabular}{cccccc}
\hline Compound & Calorimeter & $V / \mathrm{cm}^{3}$ & $m / \mathrm{g}$ & $p /$ Torr & $\rho / \mathrm{g} \mathrm{cm}^{-3}$ \\
\hline \multicolumn{7}{c}{ Low-temperature Cryostat, Mark II } \\
CoWO $_{4}$ & W-52 & 59.11 & 89.1043 & 63.0 & $7.76^{\circ}$ \\
NiWO $_{4}$ & W-30 & 18.88 & 43.7491 & 75.5 & 7.59 \\
& \multicolumn{7}{c}{} \\
CoWO $_{4}$ & Intermediate-temperature Thermostat, Mark IV & \\
& W-22-P & 84.31 & 88.3598 & 55.0 & 7.76 \\
\hline
\end{tabular}

a Compare reference 16 


\section{Results}

\section{HEAT CAPACITIES}

The heat capacities of $\mathrm{CoWO}_{4}$ are given in table 5 in chronological sequence so that temperature increments used usually may be inferred from the temperature increments between adjacent determinations, and are expressed in terms of a molar mass

TABLE 5. Heat capacity of cobalt tungstate CoWO

$\left(\mathrm{cal}_{\mathrm{th}}=4.184 \mathrm{~J}\right)$

\begin{tabular}{|c|c|c|c|c|c|c|c|c|}
\hline$\frac{T}{\mathrm{~K}}$ & $\overline{\mathrm{cal}_{\mathrm{th}} \mathrm{K}^{-1} \mathrm{~mol}^{-1}}$ & $C_{p}$ & $\frac{T}{\mathrm{~K}} \quad \overline{\mathrm{c}}$ & $\frac{C_{\mathrm{p}}}{\mathrm{cal}_{\mathrm{tn}} \mathrm{K}^{-1} \mathrm{~mol}^{-1}}$ & $\frac{T}{\mathrm{~K}}$ & $C_{p}$ & $\frac{T}{\mathrm{~K}}$ & $\frac{C_{p}}{\mathrm{cal}_{\mathrm{cn}} \mathrm{K}^{-1} \mathrm{~mol}^{-1}}$ \\
\hline \multicolumn{9}{|c|}{ Mark II Cryostat } \\
\hline & \multicolumn{2}{|c|}{ Scries I } & 303.06 & 28.62 & 12.62 & 0.139 & \multicolumn{2}{|r|}{ Series VIII } \\
\hline 92.84 & \multirow{2}{*}{\multicolumn{2}{|c|}{10.76}} & 313.15 & 29.04 & 13.87 & 0.194 & \multirow{2}{*}{\multicolumn{2}{|c|}{ See below }} \\
\hline 99.55 & & & 323.14 & 29.45 & 15.25 & 0.272 & & \\
\hline 113.17 & \multicolumn{2}{|c|}{13.58} & 333.14 & 29.85 & 16.88 & 0.371 & \multicolumn{2}{|r|}{ Series IX } \\
\hline 121.92 & \multirow{2}{*}{\multicolumn{2}{|c|}{$\begin{array}{l}14.70 \\
15.71\end{array}$}} & 343.28 & 30.33 & 18.68 & 0.516 & 50.98 & 4.435 \\
\hline 130.14 & & & \multicolumn{2}{|r|}{. } & \multirow{2}{*}{\multicolumn{2}{|c|}{ Series V }} & 52.17 & 4.529 \\
\hline 139.65 & \multirow{2}{*}{\multicolumn{2}{|c|}{16.82}} & \multicolumn{2}{|r|}{ Series III } & & & 53.32 & 4.682 \\
\hline 150.35 & & & 53.33 & 4.676 & 20.25 & 0.668 & 54.43 & 4.866 \\
\hline 160.55 & \multicolumn{2}{|c|}{19.01} & 56.45 & 5.082 & 22.37 & 0.911 & 55.49 & 4.964 \\
\hline 170.33 & \multicolumn{2}{|c|}{19.96} & 61.08 & 5.795 & 24.52 & 1.208 & 56.52 & 5.093 \\
\hline 179.79 & \multirow{2}{*}{\multicolumn{2}{|c|}{$\begin{array}{l}20.79 \\
21.65\end{array}$}} & 66.75 & 6.689 & 26.92 & 1.576 & 57.52 & 5.245 \\
\hline 189.97 & & & 73.06 & 7.679 & 29.64 & 2.054 & 58.91 & 5.445 \\
\hline 200.84 & \multicolumn{2}{|c|}{22.52} & 80.33 & 8.653 & 32.91 & 2.714 & 60.67 & 5.728 \\
\hline 21141 & \multicolumn{2}{|c|}{23.31} & \multirow{2}{*}{\multicolumn{2}{|c|}{ Series IV }} & 37.15 & 3.694 & 62.67 & 6.044 \\
\hline 221.73 & \multirow{2}{*}{\multicolumn{2}{|c|}{$\begin{array}{l}24.05 \\
24.71\end{array}$}} & & & 41.43 & 4.838 & 64.88 & 6.388 \\
\hline 231.81 & & & 4.885 & 0.008 & \multirow{2}{*}{\multicolumn{2}{|c|}{ Series VI }} & & \\
\hline & & & 5.652 & 0.010 & & & & Series X \\
\hline & Series & & 6.329 & 0.011 & & See below & 151.10 & 18.04 \\
\hline 241.58 & & 5.35 & 7.042 & 0.014 & & & 157.22 & 18.66 \\
\hline 252.13 & & 5.97 & 7.847 & 0.021 & & Series VII & 162.21 & 19.15 \\
\hline 262.48 & & 6.59 & 8.626 & 0.032 & 34.22 & 2.996 & 163.97 & 19.31 \\
\hline 272.67 & & 7.13 & 9.452 & 0.051 & 37.23 & 3.711 & 167.87 & 19.69 \\
\hline 282.72 & & 7.67 & 10.40 & 0.075 & 40.32 & 4.524 & 171.70 & 20.08 \\
\hline 292.84 & & 8.16 & 11.46 & 0.097 & 42.91 & 5.303 & 176.42 & 20.47 \\
\hline$T$ & $\Delta T$ & $\left\langle C_{p}\right\rangle$ & & $C_{p}$ & $T$ & $\Delta T$ & $\left\langle C_{p}\right\rangle$ & $C_{p}$ \\
\hline$\overline{\mathrm{K}}$ & $\overline{\mathrm{K}}$ & $\mathrm{cal}_{\mathrm{tn}} \mathrm{K}^{-1} \mathrm{r}$ & $\overline{\mathrm{mol}^{-1}} \overline{\mathrm{cal}}$ & $\overline{\mathrm{al}_{\mathrm{th}} \mathrm{K}^{-1} \mathrm{~mol}^{-1}}$ & $\overline{\mathbf{K}}$ & $\overline{\mathrm{K}} \overline{\mathrm{cal}_{\mathrm{th}} \mathbf{K}}$ & $\mathrm{K}^{-1} \mathrm{~mol}^{-1}$ & $1 \mathrm{cal}_{\mathrm{th}} \mathrm{K}^{-1} \mathrm{~mol}^{-1}$ \\
\hline & & Series & $\mathrm{V}$ & & & & eries XII & \\
\hline 45.50 & 4.42 & 6.42 & & 6.332 & 43.31 & 0.87 & 5.432 & 5.438 \\
\hline 50.95 & 6.48 & 4.71 & & 4.442 & 44.17 & 0.83 & 5.738 & 5.742 \\
\hline & & & & & 44.98 & 0.79 & 6.052 & 6.081 \\
\hline & & Series & VIII & & 45.75 & 0.75 & 6.469 & 6.454 \\
\hline 45.16 & 2.09 & 6.17 & & 6.170 & 46.23 & 0.21 & 6.608 & 6.703 \\
\hline 47.12 & 1.84 & 7.14 & & 7.302 & 46.44 & 0.20 & 6.644 & 6.831 \\
\hline 49.21 & 2.34 & 4.66 & & 4.482 & 46.64 & $4 \quad 0.20$ & 7.055 & 6.954 \\
\hline & & & & & 46.84 & 0.19 & 7.189 & 7.094 \\
\hline & & Series & XI & & 47.03 & 0.19 & 7.182 & 7.238 \\
\hline & & Enthalpy & $\operatorname{detn} . \mathbf{A}$ & & 47.22 & 0.19 & 7.476 & 7.396 \\
\hline 55.043 & 0.28 & 4.81 & & 4.813 & 47.41 & 0.19 & 7.520 & 7.562 \\
\hline
\end{tabular}


TABLE 5-continued




of $306.7808 \mathrm{~g} \mathrm{~mol}^{-1}$; those for $\mathrm{NiWO}_{4}$ are similarly given in table 6 and are expressed in terms of a molar mass of $306.5576 \mathrm{~g} \mathrm{~mol}^{-1}$; the enthalpy determinations for both compounds are summarized in table 7 . The molar heat capacities for both compounds together with revised values from the heat capacity of $\mathrm{ZnWO}_{4}$ are presented in figure $2(5$ to $350 \mathrm{~K}$ ) and figure $3(300$ to $550 \mathrm{~K})$.

TABLE 6. Heat capacity of nickel tungstate $\mathrm{NiWO}_{4}$

$\left(\mathrm{cal}_{\mathrm{th}}=4.184 \mathrm{~J}\right)$

\begin{tabular}{|c|c|c|c|c|c|c|c|c|}
\hline$\frac{T}{\mathrm{~K}}$ & \multicolumn{2}{|c|}{$C_{p}$} & $\frac{T}{\bar{K}}$ & $\frac{C_{\mathrm{p}}}{\mathrm{cal}_{\mathrm{tn}} \mathrm{K}^{-1} \mathrm{~mol}^{-1}}$ & $\frac{T}{\mathrm{~K}}$ & $C_{p}$ & $\frac{T}{\mathrm{~K}}$ & $\frac{C_{p}}{\mathrm{cal}_{\mathrm{th}} \mathrm{K}^{-1} \mathrm{~mol}^{-1}}$ \\
\hline \multicolumn{9}{|c|}{ Mark II Cryostat } \\
\hline & \multicolumn{2}{|c|}{ Series I } & 246.57 & 24.89 & \multicolumn{2}{|c|}{ Series $\mathrm{X}$} & \multirow{2}{*}{$\begin{array}{l}33.141 \\
37.062\end{array}$} & \multirow{2}{*}{$\begin{array}{l}2.235 \\
2.971\end{array}$} \\
\hline 107.22 & & 1.42 & 256.71 & 25.50 & 6.348 & 0.013 & & \\
\hline 113.83 & & 2.30 & 266.67 & 26.10 & 7.468 & 0.060 & & 3.713 \\
\hline 123.38 & & 3.51 & 276.48 & 26.66 & 7.986 & 0.043 & 45.809 & 4.686 \\
\hline 133.41 & & 4.72 & 286.14 & 27.17 & 8.748 & 0.073 & 50.340 & 5.755 \\
\hline 143.70 & & 5.94 & 295.90 & 27.66 & 9.783 & 0.082 & 52.926 & 6.426 \\
\hline 153.47 & & 7.00 & 305.74 & 28.11 & 10.998 & \multirow{2}{*}{$\begin{array}{l}0.093 \\
0.123\end{array}$} & \multicolumn{2}{|c|}{ Enthalpy detn. C } \\
\hline 163.32 & \multirow{2}{*}{\multicolumn{2}{|c|}{$\begin{array}{l}18.03 \\
19.09\end{array}$}} & \multirow{2}{*}{\multicolumn{2}{|c|}{ Series III }} & 12.030 & & \multicolumn{2}{|c|}{$66.647 \quad 6.288$} \\
\hline 173.85 & & & & & 13.057 & $\begin{array}{l}0.123 \\
0.169\end{array}$ & \multirow{2}{*}{\multicolumn{2}{|c|}{ Series XI }} \\
\hline 184.59 & & .08 & $\begin{array}{l}65.29 \\
68.81\end{array}$ & $\begin{array}{l}6.156 \\
6.548\end{array}$ & 14.130 & \multirow{2}{*}{$\begin{array}{l}0.232 \\
0.300\end{array}$} & & \\
\hline 194.98 & & .99 & $\begin{array}{l}75.44 \\
70.01\end{array}$ & 7.299 & 15.439 & & \multirow{2}{*}{$\begin{array}{l}293.85 \\
303.90\end{array}$} & \multirow{2}{*}{$\begin{array}{l}27.58 \\
28.07\end{array}$} \\
\hline 205.07 & & .83 & $\begin{array}{l}73.44 \\
83.21\end{array}$ & 8.361 & 16.877 & 0.391 & & \\
\hline 214.91 & & .61 & 92.07 & 9.540 & 18.468 & \multirow{2}{*}{$\begin{array}{l}0.511 \\
0.666\end{array}$} & 314.22 & 28.53 \\
\hline 224.95 & & .36 & $\begin{array}{r}92.07 \\
102.26\end{array}$ & 10.86 & 20.224 & & 324.44 & 28.95 \\
\hline 235.21 & & .08 & 112.65 & 12.20 & 22.145 & \multirow{2}{*}{$\begin{array}{l}0.851 \\
1.113\end{array}$} & 334.54 & 29.41 \\
\hline \multirow{2}{*}{\multicolumn{3}{|c|}{ Series II }} & \multirow{3}{*}{\multicolumn{2}{|c|}{$\begin{array}{l}\text { Series IV to IX } \\
\text { See below }\end{array}$}} & 24.408 & & 344.54 & 29.82 \\
\hline & & & & & 26.850 & \multirow{2}{*}{\multicolumn{3}{|c|}{$\begin{array}{l}1.424 \\
1.809\end{array}$}} \\
\hline 236.40 & & .15 & & & 29.598 & & & \\
\hline$T$ & $\Delta T$ & $\left\langle C_{p}\right\rangle$ & & $C_{p}$ & $T$ & $\Delta T$ & $\left\langle C_{p}\right\rangle$ & $C_{p}$ \\
\hline$\overline{\mathbf{K}}$ & $\overline{\mathbf{K}}$ & $\mathrm{cal}_{\mathrm{th}} \mathrm{K}^{-1} \mathrm{n}$ & $\mathrm{mol}^{-1}$ & $\overline{\mathrm{lth}} \mathrm{K}^{-1} \mathrm{~mol}^{1}$ & $\overline{\mathbf{K}}$ & $\overline{\mathrm{K}} \overline{\mathrm{cal}_{\mathrm{t}}}$ & in $\mathrm{K}^{-1} \mathrm{~mol}^{-1}$ & $\mathrm{cal}_{\mathrm{th}} \mathrm{K}^{-1} \mathrm{~mol}^{-1}$ \\
\hline & & Series & & & 60.538 & 0.261 & 6.541 & 6.540 \\
\hline 51.755 & 2.070 & 6.138 & & 6.132 & 60.809 & 0.265 & 6.269 & 6.256 \\
\hline 53.754 & 1.918 & 6.683 & & 6.683 & 61.083 & 0.267 & 6.140 & 6.156 \\
\hline 55.694 & 1.949 & 7.276 & & 7.276 & 61.468 & 0.487 & 6.092 & 6.090 \\
\hline 57.580 & 1.809 & 7.963 & & 7.958 & 62.090 & 0.749 & 6.056 & 6.066 \\
\hline 59.326 & 1.672 & 8.831 & & 8.896 & & & & \\
\hline 61.141 & 1.947 & 6.343 & & 6.146 & & & Series VI & \\
\hline 63.091 & 1.938 & 6.082 & & 6.078 & 55.464 & 1.937 & 7.194 & 7.200 \\
\hline & & & & & 57.338 & 1.801 & & 7.854 \\
\hline & & Series & & & 58.415 & 0.339 & 8.320 & 8.309 \\
\hline 52.097 & 2.186 & 6.214 & & 6.231 & 58.757 & 0.334 & 8.501 & 8.483 \\
\hline 54.203 & 2.014 & 6.809 & & 6.820 & 59.032 & 0.205 & 8.631 & 8.631 \\
\hline 56.153 & 1.872 & 7.412 & & 7.428 & 59.240 & 0.202 & 8.788 & 8.811 \\
\hline 57.970 & 1.750 & 8.141 & & 8.118 & 59.446 & 0.199 & 9.047 & 9.050 \\
\hline 59.016 & 0.325 & 8.658 & & 8.636 & 59.649 & 0.196 & 9.243 & 9.240 \\
\hline 59.344 & 0.318 & 8.904 & & 8.906 & 59.850 & 0.195 & 9.294 & - \\
\hline 59.664 & 0.311 & 9.221 & & 9.256 & 60.059 & 0.205 & 8.440 & 8.281 \\
\hline 59.984 & 0.317 & 8.868 & & 8.860 & 60.279 & 0.223 & 7.170 & 7.082 \\
\hline 60.275 & 0.249 & 7.200 & & 7.080 & 60.514 & 0.232 & 6.597 & 6.524 \\
\hline
\end{tabular}


TABLE 6-continued



TABLE 7. Enthalpy determinations of cobalt and nickel tungstates, $\mathrm{CoWO}_{4}$ and $\mathrm{NiWO}_{4}$, in the Mark II cryostat

$\left(\mathrm{cal}_{\mathrm{th}}=4.184 \mathrm{~J}\right)$

\begin{tabular}{|c|c|c|c|c|}
\hline Determination & $\frac{T_{1}}{\mathrm{~K}}$ & $\frac{T_{2}}{\mathrm{~K}}$ & $\frac{H\left(T_{2}\right)-H\left(T_{1}\right)}{\mathrm{cal}_{\mathrm{th}} \mathrm{mol}^{-1}}$ & $\frac{H(55 \mathrm{~K})-H(35 \mathrm{~K})}{\mathrm{cal}_{\mathrm{Eb}} \mathrm{mol}^{-1}}$ \\
\hline \multicolumn{5}{|c|}{ CoWO $_{4}$ in Mark II Cryostat } \\
\hline $\begin{array}{l}\text { A (Series XI) } \\
\text { B (Series XV) } \\
\text { Series VII, VIII, and IX } \\
\text { Series XII }\end{array}$ & $\begin{array}{l}32.66 \\
35.88 \\
35.54 \\
42.88\end{array}$ & $\begin{array}{l}54.90 \\
54.55 \\
54.97 \\
51.11\end{array}$ & $\begin{array}{r}104.43 \\
92.96 \\
96.04 \\
46.48\end{array}$ & $\begin{array}{l}98.10 \\
98.02 \\
98.04 \\
98.04\end{array}$ \\
\hline & & & \multicolumn{2}{|c|}{ Mean: } \\
\hline Determination & $\frac{T_{1}}{\mathbf{K}}$ & $\frac{T_{2}}{K}$ & \multicolumn{2}{|c|}{$\frac{H\left(T_{2}\right)-H\left(T_{1}\right)}{\mathrm{cal}_{\mathrm{th}} \mathrm{mol}^{-1}} \frac{H(65 \mathrm{~K})-H(45 \mathrm{~K})}{\mathrm{cal}_{\mathrm{tb}} \mathrm{mol}^{-1}}$} \\
\hline \multicolumn{5}{|c|}{ NiWO $_{4}$ in Mark II Cryostat } \\
\hline $\begin{array}{l}\text { A (Series VII) } \\
\text { B (Series IX) } \\
\text { C (Series X) } \\
\text { Series V }\end{array}$ & $\begin{array}{l}54.78 \\
52.28 \\
53.37 \\
51.00\end{array}$ & $\begin{array}{l}64.16 \\
65.02 \\
66.03 \\
62.47\end{array}$ & $\begin{array}{l}67.88 \\
89.85 \\
88.82 \\
81.681\end{array}$ & $\begin{array}{l}128.93 \\
128.94 \\
128.68 \\
128.65\end{array}$ \\
\hline & & & \multicolumn{2}{|c|}{ Mean: $(128.80 \pm 0.28)$} \\
\hline
\end{tabular}




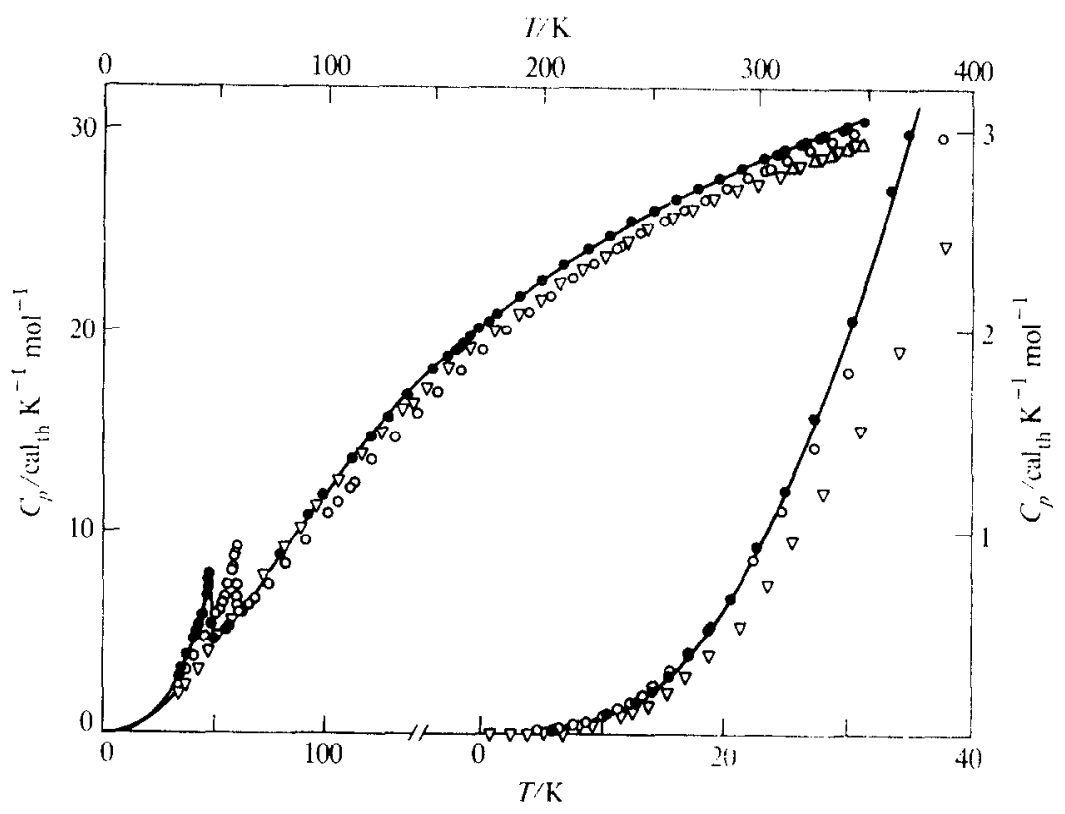

FIGURE 2. Heat capacities of zinc, nickel, and cobalt tungstates, 5 to $350 \mathrm{~K}$., $\mathrm{CoWO}_{4}$ (Mark II results); $\mathrm{O}, \mathrm{NiWO}_{4}$ (Mark II results); $\triangle, \mathrm{ZnWO}_{4}$ (Mark II results); $\Lambda, \mathrm{ZnWO}_{4}$ (Mark IV results).

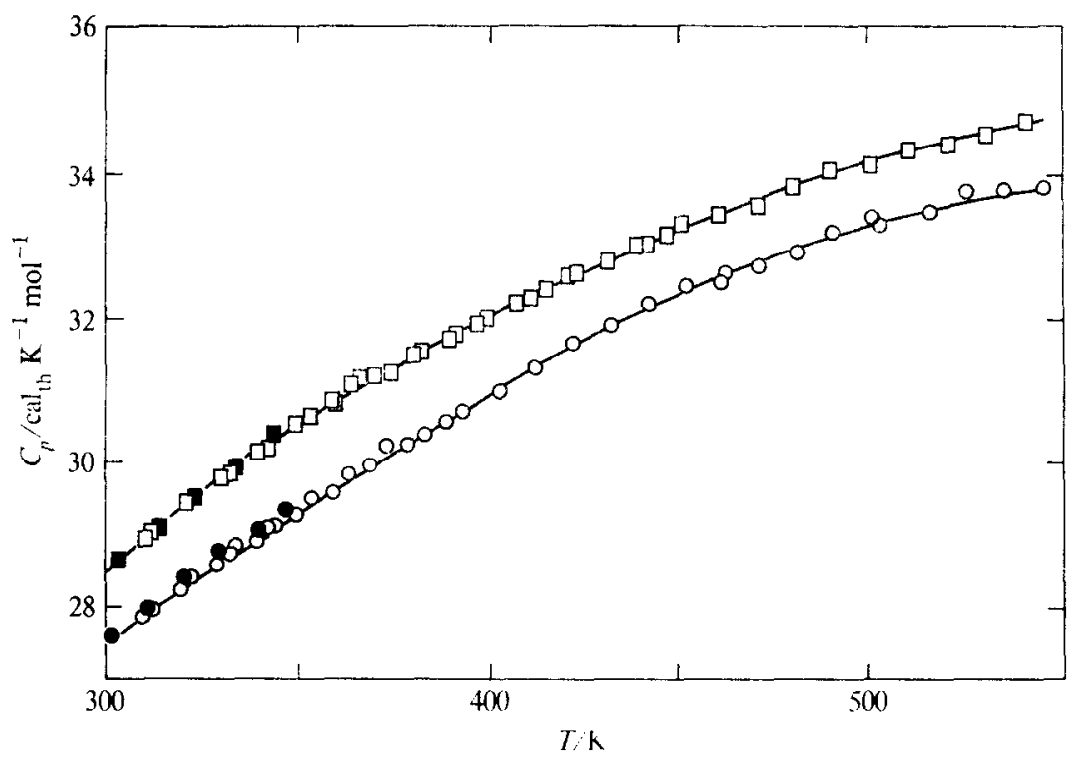

FIGURE 3. Heat capacities of zinc and cobalt tungstates, 300 to $550 \mathrm{~K}$. 口, CoWO, (Mark II results); $\square$, CoWO (Mark IV results);, $\mathrm{ZnWO}_{4}$ (Mark II results); O, ZnWO (Mark IV results). 


\section{ADJUSTMENT FOR IMPURITIES}

The presence of the separate phase impurities in the nickel tungstate necessitated adjustment of the results in terms of the enthalpies of $\alpha$-cristobalite and calcium tungstate. The thermal functions for silicon dioxide used in the analysis were generated by curve fitting the low-temperature, 5 to $300 \mathrm{~K}$, heat capacities of Westrum ${ }^{(23)}$ which had been extrapolated to $350 \mathrm{~K}$. The adjustment for $\mathrm{SiO}_{2}$ amounted to about 1 per cent below $10 \mathrm{~K}$ and about 0.5 per cent over the rest of the temperature region measured. The thermal functions for calcium tungstate used in the analysis for nickel tungstate were generated from the polynomial representation of the low-temperature heat capacities of Lyon and Westrum. ${ }^{(24)}$ The adjustment for separate-phase calcium tungstate was less than 0.1 per cent of the molar heat capacity. Since the mole percentage of the sodium tungstate impurity phase was so low and since data for this compound are available only above $50 \mathrm{~K},{ }^{(25)}$ no adjustment was made to the heat capacity of nickel tungstate for the sodium tungstate phase. Preliminary corrections showed the correction for sodium tungstate to amount to less than $\mathbf{0 . 0 5}$ per cent in regions where reasonable estimates could be made for the heat capacity of sodium tungstate.

\section{THERMOPHYSICAL FUNCTIONS}

The experimental molar heat capacities for both samples in non-transition regions (nickel tungstate adjusted for impurities) were fitted to polynomials in temperature by the method of least squares, and integrated to yield values of the thermophysical functions at selected temperature intervals. These derived values are presented in tables 8 and 9 for cobalt and nickel tungstates. Within the transition regions the thermal functions are based upon numerical integration of heat-capacity points mapped on large scale plots.

TABLE 8. Thermophysical functions of cobalt tungstate CoWO $\left(\mathrm{cal}_{\mathrm{th}}=4.184 \mathrm{~J}\right)$

\begin{tabular}{|c|c|c|c|c|}
\hline$\frac{T}{\mathrm{~K}}$ & $\frac{C_{p}}{\mathrm{cal}_{\mathrm{th}} \mathrm{K}^{-1} \mathrm{~mol}^{-1}}$ & $\frac{\left\{S^{\circ}(T)-S^{\circ}(0)\right\}}{\mathrm{cal}_{\mathrm{th}} \mathrm{K}^{-1} \mathrm{~mol}^{-1}}$ & $\frac{H^{\circ}(T)-H^{\circ}(0)}{\mathrm{cal}_{\mathrm{tb}} \mathrm{mol}^{-1}}$ & $\frac{-\left\{G^{\circ}(T)-H^{\circ}(0)\right\} / T}{\text { cal }_{\text {th }} \mathrm{K}^{-1} \mathrm{~mol}^{-1}}$ \\
\hline 5 & 0.005 & $(0.003)$ & $(0.012)$ & $(0.001)$ \\
\hline 10 & 0.061 & 0.016 & 0.122 & 0.004 \\
\hline 15 & 0.253 & 0.072 & 0.843 & 0.016 \\
\hline 20 & 0.642 & 0.193 & 2.982 & 0.044 \\
\hline 25 & 1.275 & 0.400 & 7.676 & 0.093 \\
\hline 30 & 2.124 & 0.704 & 16.09 & 0.168 \\
\hline 35 & 3.176 & 1.108 & 29.25 & 0.272 \\
\hline 40 & 4.435 & 1.612 & 48.19 & 0.407 \\
\hline 45 & 6.094 & 2.223 & 74.20 & \\
\hline 50 & 4.380 & 2.858 & 104.24 & \\
\hline 60 & 5.623 & 3.752 & 153.31 & 1.194 \\
\hline 70 & 7.189 & 4.737 & 217.58 & 1.628 \\
\hline 80 & 8.783 & 5.800 & 297.39 & 2.083 \\
\hline 90 & 10.328 & 6.925 & 393.02 & 2.558 \\
\hline 100 & 11.790 & 8.089 & 503.7 & 3.052 \\
\hline
\end{tabular}


TABLE 8-continued

\begin{tabular}{|c|c|c|c|c|}
\hline$\frac{T}{\mathrm{~K}}$ & $\frac{C_{p}}{\mathrm{cal}_{\mathrm{th}} \mathrm{K}^{-1} \mathrm{~mol}^{-1}}$ & $\frac{\left\{S^{\circ}(T)-S^{\circ}(0)\right\}}{\mathrm{cal}_{\mathrm{ED}} \mathrm{K}^{-1} \mathrm{~mol}^{-1}}$ & $\frac{H^{\circ}(T)-H^{\circ}(0)}{\mathrm{cal}_{\mathrm{tn}} \mathrm{mol}^{-1}}$ & $\frac{-\left\{G^{\circ}(T)-H^{\circ}(0)\right\} / T}{\mathrm{cal}_{\mathrm{th}} \mathrm{K}^{-1} \mathrm{~mol}^{-1}}$ \\
\hline $\begin{array}{l}110 \\
120 \\
130 \\
140 \\
150\end{array}$ & $\begin{array}{l}13.170 \\
14.471 \\
15.69 \\
16.85 \\
17.93\end{array}$ & $\begin{array}{r}9.278 \\
10.481 \\
11.688 \\
12.893 \\
14.093\end{array}$ & $\begin{array}{r}628.6 \\
766.8 \\
917.7 \\
1080.5 \\
1254.4\end{array}$ & $\begin{array}{l}3.564 \\
4.090 \\
4.628 \\
5.176 \\
5.730\end{array}$ \\
\hline $\begin{array}{l}160 \\
170 \\
180 \\
190 \\
200\end{array}$ & $\begin{array}{l}18.94 \\
19.90 \\
20.80 \\
21.65 \\
22.46\end{array}$ & $\begin{array}{l}15.28 \\
16.46 \\
17.62 \\
18.77 \\
19.90\end{array}$ & $\begin{array}{l}1438.8 \\
1633.1 \\
1836.7 \\
2049.0 \\
2269.6\end{array}$ & $\begin{array}{l}6.290 \\
6.854 \\
7.420 \\
7.987 \\
8.555\end{array}$ \\
\hline $\begin{array}{l}210 \\
220 \\
230 \\
240 \\
250\end{array}$ & $\begin{array}{l}23.21 \\
23.93 \\
24.61 \\
25.25 \\
25.86\end{array}$ & $\begin{array}{l}21.02 \\
22.11 \\
23.19 \\
24.25 \\
25.30\end{array}$ & $\begin{array}{l}2498.0 \\
2733.7 \\
2976.4 \\
3225.7 \\
3481.3\end{array}$ & $\begin{array}{r}9.122 \\
9.687 \\
10.251 \\
10.813 \\
11.371\end{array}$ \\
\hline $\begin{array}{l}260 \\
270 \\
280 \\
290 \\
300\end{array}$ & $\begin{array}{l}26.44 \\
26.99 \\
27.52 \\
28.02 \\
28.50\end{array}$ & $\begin{array}{l}26.32 \\
27.33 \\
28.32 \\
29.30 \\
30.25\end{array}$ & $\begin{array}{l}3742.8 \\
4010.0 \\
4282.5 \\
4560.2 \\
4842.8\end{array}$ & $\begin{array}{l}11.926 \\
12.478 \\
13.026 \\
13.571 \\
14.111\end{array}$ \\
\hline $\begin{array}{l}310 \\
320 \\
330 \\
340 \\
350\end{array}$ & $\begin{array}{l}28.95 \\
29.38 \\
29.78 \\
30.16 \\
30.52\end{array}$ & $\begin{array}{l}31.20 \\
32.12 \\
33.03 \\
33.93 \\
34.81\end{array}$ & $\begin{array}{l}5130 \\
5422 \\
5718 \\
6017 \\
6321\end{array}$ & $\begin{array}{l}14.647 \\
15.18 \\
15.71 \\
16.23 \\
16.75\end{array}$ \\
\hline $\begin{array}{l}360 \\
370 \\
380 \\
390 \\
400\end{array}$ & $\begin{array}{l}30.86 \\
31.17 \\
31.47 \\
31.75 \\
32.02\end{array}$ & $\begin{array}{l}35.67 \\
37.52 \\
37.36 \\
38.18 \\
38.98\end{array}$ & $\begin{array}{l}6628 \\
6938 \\
7251 \\
7567 \\
7886\end{array}$ & $\begin{array}{l}17.26 \\
17.77 \\
18.27 \\
18.77 \\
19.27\end{array}$ \\
\hline $\begin{array}{l}410 \\
420 \\
430 \\
440 \\
450\end{array}$ & $\begin{array}{l}32.27 \\
32.52 \\
32.75 \\
32.98 \\
33.20\end{array}$ & $\begin{array}{l}39.78 \\
40.56 \\
41.33 \\
42.08 \\
42.83\end{array}$ & $\begin{array}{l}8208 \\
8531 \\
8858 \\
9187 \\
9517\end{array}$ & $\begin{array}{l}19.76 \\
20.25 \\
20.73 \\
21.20 \\
21.68\end{array}$ \\
\hline $\begin{array}{l}460 \\
470 \\
480 \\
490 \\
500\end{array}$ & $\begin{array}{l}33.41 \\
33.61 \\
33.80 \\
33.97 \\
34.12\end{array}$ & $\begin{array}{l}43.56 \\
44.28 \\
44.99 \\
45.69 \\
46.37\end{array}$ & $\begin{array}{r}9851 \\
10186 \\
10523 \\
10862 \\
11202\end{array}$ & $\begin{array}{l}22.14 \\
22.61 \\
23.07 \\
23.52 \\
23.97\end{array}$ \\
\hline $\begin{array}{l}510 \\
520 \\
530 \\
540 \\
550\end{array}$ & $\begin{array}{l}34.26 \\
34.40 \\
34.53 \\
34.68 \\
34.82\end{array}$ & $\begin{array}{l}47.05 \\
47.72 \\
48.38 \\
49.02 \\
49.66\end{array}$ & $\begin{array}{l}11544 \\
11887 \\
12232 \\
12578 \\
12925\end{array}$ & $\begin{array}{l}24.42 \\
24.86 \\
25.30 \\
25.73 \\
26.16\end{array}$ \\
\hline 273.15 & 27.16 & 27.64 & 4095.2 & 12.651 \\
\hline 298.15 & 28.41 & 30.08 & 4790.2 & 14.011 \\
\hline
\end{tabular}


TABLE 9. Thermophysical functions of nickel tungstate $\mathrm{NiWO}_{4}$ $\left(\mathrm{Cal}_{\mathrm{kb}}=4.184 \mathrm{~J}\right)$

\begin{tabular}{|c|c|c|c|c|}
\hline$\frac{T}{\mathbf{K}}$ & $\frac{C_{\mathrm{p}}}{\mathrm{Cal}_{\mathrm{ln}} \mathrm{K}^{-1} \mathrm{~mol}^{-1}}$ & $\frac{\left\{S^{\circ}(T)-S^{\circ}(0)\right\}}{\mathrm{cal}_{\mathrm{kn}} \mathrm{K}^{-1} \mathrm{~mol}^{-1}}$ & $\frac{H^{\circ}(T)-H^{\circ}(0)}{\mathrm{cal}_{\mathrm{th}} \mathrm{mol}^{-1}}$ & $\frac{-\left\{G^{\circ}(T)-H^{\circ}(0)\right\} / T}{\mathrm{cal}_{\mathrm{th}} \mathrm{K}^{-1} \mathrm{~mol}^{-1}}$ \\
\hline 5 & 0.010 & $(0.003)$ & $(0.012)$ & $(0.001)$ \\
\hline 10 & 0.079 & 0.026 & 0.198 & 0.007 \\
\hline 15 & 0.273 & 0.090 & 1.014 & 0.022 \\
\hline 20 & 0.643 & 0.215 & 3.228 & 0.053 \\
\hline 25 & 1.187 & 0.414 & 7.739 & 0.104 \\
\hline 30 & 1.861 & 0.688 & 15.32 & 0.178 \\
\hline 35 & 2.628 & 1.032 & 26.50 & 0.274 \\
\hline 40 & 3.497 & 1.438 & 41.77 & 0.394 \\
\hline 45 & 4.505 & 1.907 & 61.71 & 0.535 \\
\hline 50 & 5.672 & 2.440 & 87.08 & 0.699 \\
\hline 60 & 8.604 & 3.740 & 158.8 & \\
\hline 70 & 6.655 & 4.717 & 222.2 & 1.541 \\
\hline 80 & 7.886 & 5.683 & 294.7 & 1.998 \\
\hline 90 & 9.241 & 6.690 & 380.3 & 2.463 \\
\hline 100 & 10.568 & 7.733 & 479.4 & 2.938 \\
\hline 110 & 11.843 & 8.800 & 591.6 & 3.422 \\
\hline 120 & 13.079 & 9.884 & 716.2 & 3.915 \\
\hline 130 & 14.289 & 10.978 & 853.1 & 4.416 \\
\hline 140 & 15.47 & 12.081 & 1001.9 & 4.925 \\
\hline 150 & 16.61 & 13.188 & 1162.4 & 5.438 \\
\hline 160 & 17.70 & 14.295 & 1334.0 & 5.957 \\
\hline 170 & 18.73 & 15.40 & 1516.2 & 6.480 \\
\hline 180 & 19.68 & 16.50 & 1708.3 & 7.006 \\
\hline 190 & 20.57 & 17.59 & 1909.7 & 7.535 \\
\hline 200 & 21.41 & 18.66 & 2119.7 & 8.064 \\
\hline 210 & 22.21 & 19.73 & 2337.8 & 8.594 \\
\hline 220 & 22.97 & 20.78 & 2563.7 & 9.124 \\
\hline 230 & 23.70 & 21.81 & 2797.1 & 9.653 \\
\hline 240 & 24.41 & 22.84 & 3037.7 & 10.181 \\
\hline 250 & 25.09 & 23.85 & 3285.2 & 10.708 \\
\hline 260 & 25.72 & 24.85 & 3539.3 & 11.233 \\
\hline 270 & 26.31 & 25.83 & 3799.5 & 11.755 \\
\hline 280 & 26.86 & 26.79 & 4065.4 & 12.275 \\
\hline 290 & 27.37 & 27.75 & 4336.6 & 12.792 \\
\hline 300 & 27.86 & 28.68 & 4612.8 & 13.306 \\
\hline 310 & 28.34 & 29.60 & 4893.7 & 13.817 \\
\hline 320 & 28.81 & 30.51 & 5179 & 14.325 \\
\hline 330 & 29.27 & 31.40 & 5470 & 14.829 \\
\hline 340 & 29.71 & 32.28 & 5765 & 15.33 \\
\hline 350 & 30.13 & 33.15 & 6064 & 15.83 \\
\hline 273.15 & 26.49 & 26.13 & 3882.7 & 11.919 \\
\hline 298.15 & 27.77 & 28.51 & 4561.3 & 13.211 \\
\hline
\end{tabular}


The estimated error in the thermodynamic functions of cobalt tungstate is less than 0.1 per cent above $100 \mathrm{~K}$. The error for the thermodynamic functions of nickel tungstate is larger, due to the uncertainty in the mass fractions of the impurity phases. Since the accumulative correction to the molar heat capacity is less than 0.6 per cent, the uncertainty in the thermal functions is still less than 0.2 per cent above $100 \mathrm{~K}$.

The values of the entropy and enthalpy at the lowest temperatures in parentheses in the tables of thermal functions were obtained from plots of $C_{p} / T$ against $T^{2}$. For either compound, the magnitude of this extrapolation is negligible. The entropies and Gibbs energies have not been adjusted for nuclear spin or for isotopic mixing contributions and, hence, are practical values for use in chemical thermodynamic calculations.

\section{Discussion}

\section{SUPEREXCHANGE INTERACTIONS}

As a consequence of the intensive study of magnetic interactions in insulators, semiempirical rules, the Goodenough-Kanamori rules (clearly formulated in reviews by Anderson) ${ }^{(26,27)}$ have been developed for predicting the sign of the interaction through which magnetic ions couple their moments. When cations occupy octahedral holes in closest-packed anion lattices, e.g. for tungstates and dichlorides, ${ }^{(28)}$ the cation-anion-cation angle is $90^{\circ}$ and the Goodenough-Kanamori rules predict antiferromagnetic exchange for the iron(II), cobalt, and nickel compounds (compare Goodenough ${ }^{(29)}$ ). The iron(II), cobalt, and nickel tungstates (and dichlorides) are antiferromagnets in which the spins are ferromagnetically aligned within each layer and the spins in neighboring layers are aligned antiferromagnetically. ${ }^{(30)}$ In $\mathrm{MnWO}_{4}$ (and $\mathrm{MnCl}_{2}$ ), however, the cations do not order ferromagnetically ${ }^{(31)}$ within each layer.

Detailed superexchange paths in the tungstates were first discussed by Van Uitert et al. ${ }^{(3)}$ The structure of the tungstates can be thought of as a (distorted) hexagonal closest-packed array of oxygens with the tungsten and metal ions in the octahedral holes, forming independent zigzag chains along the $c$-axis of the crystal. ${ }^{(32)}$ Each $\mathrm{W}$-octahedral chain is attached by common corners to four $\mathrm{M}$-chains and each M-chain is also surrounded by four W-chains. Each $\mathrm{M}^{2+}$ ion can interact by $90^{\circ}$ superexchange with its two nearest neighbors in the chain, with which it shares two oxygen atoms each. There is also the possibility of next-nearest neighbor exchange within the chains, involving the tungsten atoms in an adjacent octahedron. Interchain coupling is possible both between layers and within the same layer by exchange paths of the type $\mathrm{M}-\mathrm{O}-\mathrm{W}-\mathrm{O}-\mathrm{M}$, where all the angles involved are close to $90^{\circ}$. These interlayer interactions should be large, since the results of Lesne and Caillet ${ }^{(33)}$ predict that the strongest bonding paths are between layers. Since the exchange interaction is enhanced with bond strength, the interlayer exchange interaction should be significant. Superexchange interactions of the type M-O-W-O-M have been studied in the ordered perovskites by Blasse ${ }^{(34)}$ and found to be comparable in strength to those in the tungstates. 


\section{MAGNETIC BEHAVIOR}

Susceptibilities of the tungstates ${ }^{(3-5)}$ show characteristic maxima associated with antiferromagnetic phase changes. Since magnetic moments of the iron, cobalt, and nickel ions are all in excess of expectation for a spin-only model, considerable orbital angular momentum is retained by the ions within their low-symmetry crystal fields. Calculations predicting the amount retained involve lengthy considerations of the low-symmetry distortions of the octahedral fields and the magnitudes of the spin-orbit coupling. Cobalt tungstate is the only tungstate for which this calculation has been done. The authors, Zvyagin and Khats'ko, ${ }^{(9)}$ were able to calculate susceptibilities and magnetic moments from their assigned energy levels in good accord with their experimental values.

Also significant is the discrepancy between the paramagnetic Curie temperatures $\Theta_{p}$ derived from susceptibility measurements in the low-temperature region and the high-temperature $(290$ to $700 \mathrm{~K})$ measurements of Shapovalova et al. ${ }^{(4)}$ which yield positive $\Theta_{p}$ 's for ferrous and cobalt tungstate. These positive $\Theta_{p}$ 's are indicative of ferromagnetic interactions within the crystal, even though the materials are known to be antiferromagnetic. Since in cobalt and iron(II) tungstate the ions in the $b c$ plane are aligned ferromagnetically, with adjacent planes aligned antiferromagnetically, the high-temperature determination of susceptibility may reflect the dominance of the intralayer exchange. This type of behavior is known to exist in the transitionmetal dichlorides where adjacent planes of ferromagnetic ions are aligned antiferromagnetically. The strong intralayer ferromagnetic interaction leads to positive paramagnetic Curie temperatures even at low temperatures. As pointed out by Dachs, ${ }^{(12)}$ molecular-field theory leads to the relation:

$$
|\Theta|_{\mathrm{p}} / T_{\mathrm{n}}=\Sigma J_{i j} / \Sigma J_{i j} S_{i} \cdot S_{j}
$$

In $\mathrm{MnWO}_{4}$, the near-neighbor antiferromagnetic coupling leads to the cancellation of terms in the denominator, producing a large ratio (5.5). The ferromagnetic planes in the $\mathrm{FeWO}_{4}, \mathrm{CoWO}_{4}$, and $\mathrm{NiWO}_{4}$ do not produce such cancellations and the ratios are significantly lower, $(0.3,1.8$, and 0.8 , respectively).

\section{SPECIAL INFORMATION}

Several investigations have been made of the electronic energy levels of pure and of doped diamagnetic tungstates. Study of the latter was undertaken with the intention of eliminating exchange effects.

In studies on the pure tungstates, Ferguson and others ${ }^{(35,36)}$ measured the absorption spectra of $\mathrm{CoWO}_{4}$ and $\mathrm{NiWO}_{4}$.

As discussed by Lyon, ${ }^{(37)}$ the crystal-field splittings are 25 and 18 per cent less for the $\mathrm{Co}^{2+}$ and $\mathrm{Ni}^{2+}$ ions in the tungstate than when they are octahedrally coordinated in the diamagnetic oxide $\mathrm{MgO} .^{(35,36)}$ Such a decrease is sufficient to alter the expected colors of the samples. Whereas octahedrally coordinated cobalt and nickel compounds in solution are usually pink and green, the respective tungstates are blue and mustard yellow. These decreases in crystal-field splitting are attributed to the electron-withdrawing power of the $\mathrm{W}^{6+}$ ion, as mentioned by Lyon ${ }^{(37)}$ and 
also by Shannon and Vincent ${ }^{(38)}$ in their discussion of covalency in the tungstates. This large effect by the tungsten ion makes the superexchange path involving the tungsten important.

The optical absorption spectra of $\mathrm{Ni}^{2+}$ ion in $\mathrm{ZnWO}_{4}$ has been studied by Reynolds et al. ${ }^{(39)}$ In an octahedral field the ground state of $\mathrm{Ni}^{2+}$ is a singlet, which will not split under a crystal field of lower symmetry. Reynolds et al. found this ${ }^{3} \mathrm{~A}_{2}$ term to lie more than $6000 \mathrm{~cm}^{-1}$ below the first excited ${ }^{3} \mathrm{~T}_{2}(\mathrm{E})$ term. Still, the spin-orbit coupling in the nickel ion must reintroduce orbital angular momentum into the ground states, since the effective magnetic moments exceed the spin-only prediction. However, no information is available as regards these energy states.

The assignment of the lowest levels for $\mathrm{Co}^{2+}$ ions in diamagnetic tungstate matrices has been accomplished by Atsarkin et al. ${ }^{(40)}$ for $\mathrm{Co}^{2+}: \mathrm{MgWO}_{4}$, and by Zvyagin and Khats'ko, ${ }^{(41)}$ and by Bates and co-workers, ${ }^{(42)}$ for $\mathrm{Co}^{2+}: \mathrm{ZnWO}_{4}$. Atsarkin et al. measured the optical absorption spectra of the cobalt ions in $\mathrm{MgWO}_{4}(0.01$ mass per cent of $\mathrm{Co}^{2+}$ ) at $300 \mathrm{~K}$. They were able to interpret the spectra observed without considering either the orthorhombic components of the crystal field or the noncentrosymmetric location of the cobalt ions. They used the crystal-field parameters derived from the spectra to calculate the splitting of the lowest triplet state into six Kramers doublets by the spin-orbit interaction. Zvyagin and Khats'ko use the results of e.p.r. studies of $\mathrm{Co}^{2+}: \mathrm{ZnWO}_{4}$ as well as their own measurements of the singlecrystal magnetic susceptibilities to assign energy levels to the six Kramers doublets. The agreement with the results of Atsarkin et al. is reasonable. Bates et al. have also studied the e.p.r. spectrum of $\mathrm{Co}^{2+}: \mathrm{ZnWO}_{4}$ and assigned energies to the six doublets on the basis of their results. The agreement with the results of Zvyagin and Khats'ko is excellent.

An additional estimate of the wavenumber interval $\Delta$ separating the ground-state doublet from the first excited doublet of $\mathrm{Co}^{2+}$ in $\mathrm{ZnWO}_{4}$ has been given by Galkin et al. $^{(43)}$ They interpret their measurements of the broadening of the e.p.r. line in terms of spin-lattice relation times with a value $\Delta=(230 \pm 30) \mathrm{cm}^{-1}$.

\section{SCHOTTKY ANOMALIES AND MAGNETIC TRANSFORMATION}

Using the standard formulas for heat capacity as a function of energy levels, ${ }^{(44)}$ it is possible to calculate the expected Schottky-type behavior in the heat capacity of cobalt tungstate. Beginning at about $60 \mathrm{~K}$, the heat capacity should rise to a maximum of about $1.35 \mathrm{cal}_{\mathrm{th}} \mathrm{K}^{-1} \mathrm{~mol}^{-1}$ at a temperature of $200 \mathrm{~K}$, then drop 10 per cent by $300 \mathrm{~K}$, after which it should slowly rise towards the maximum as the temperature increases beyond $500 \mathrm{~K}$.

Since the ground state of nickel tungstate is an orbital singlet, the magnetic entropy to be obtained will simply be $R \ln (2 S+1)=R \ln 3$. At the transition temperature of cobalt tungstate (for which only the lowest Kramers doublet is thermally populated) the expected entropy change associated with the antiferromagnetic ordering would be $R \ln 2$ similar to the behavior of cobalt in $\mathrm{CoF}_{2}$ and $\mathrm{CoCl}_{2}{ }^{(45)}$ In addition, there should be further contributions to the entropy attributed to the population of the higher-lying doublets. 


\section{RESOLUTION OF MAGNETIC AND LATTICE CONTRIBUTIONS}

In the preliminary step, the Debye characteristic temperatures $\Theta_{\mathrm{D}}$ were calculated for each experimental point for tungstate including zinc tungstate. ${ }^{(14)}$ The curve for $\Theta_{D}$ of zinc tungstate behaves normally. The other two curves have regions where the values are anomalously low; these correspond to the regions of large magnetic contributions to the heat capacity. Ratios of the $\Theta_{\mathrm{D}}$ 's: $\Theta_{\mathrm{D}}\left(\mathrm{CoWO} \mathrm{CW}_{4}\right) / \Theta_{\mathrm{D}}\left(\mathrm{ZnWO}_{4}\right)$ and $\Theta_{\mathrm{D}}\left(\mathrm{NiWO}_{4}\right) / \Theta_{\mathrm{D}}\left(\mathrm{ZnWO}_{4}\right)$ were calculated as a function of temperature. For these compounds, the ratios below $100 \mathrm{~K}$ reflect the magnetic contributions to the heat capacity.

Assessment of the low-temperature $\Theta_{D}$, and subsequently the low-temperature heat capacity, involves a complication due to the presence of the excited state in the cobalt ion because the Schottky contributions to the heat capacity of cobalt tungstate lower the observed $\Theta_{D}$ 's. To determine the lattice heat capacity, Schottky heat capacities were generated from the spectroscopic data of $\mathrm{Co}^{2+}$ ions in $\mathrm{MgWO}_{4}$ $\left(\mathrm{Co}^{2+}: \mathrm{MgWO}_{4}\right)$ and $\mathrm{Co}^{2+}$ ions in $\mathrm{ZnWO}_{4}\left(\mathrm{Co}^{2+}: \mathrm{ZnWO}_{4}\right)$. These heat capacities were then subtracted from the experimental heat capacities and the $\Theta_{\mathrm{D}}$ 's calculated for the resulting values. Since the harmonic approximation predicts $\Theta_{\mathrm{D}}\left(\mathrm{CoWO}_{4}\right) /$ $\Theta_{\mathrm{D}}\left(\mathrm{ZnWO}_{4}\right)=\{M(\mathrm{Zn}) / M(\mathrm{Co})\}^{1 / 2}=1.053$, the estimated curve was drawn to this value as a lower limit. The molar masses of the ions rather than of the molecules were used since the heavy tungsten atoms form a matrix in which the first-row metals oscillate. Such behavior has been seen in the studies of iron impurities in metal host lattices. ${ }^{(46)}$ Additional evidence for such a limit is the valence-force-field calculation of Lesne and Caillet ${ }^{(33)}$ which showed the strongest bonds within the crystal were between $\mathrm{W}$ and $\mathrm{O}$; the metal ions are bound relatively weakly to the oxygens. Hence, at temperatures greater than $100 \mathrm{~K}$, the estimated curve was smoothly interpolated between the two calculated curves.

\section{$\mathrm{NiWO}_{4}$}

A simple mass adjustment of $\Theta_{D}$ does not suffice for a lattice estimate of nickel tungstate. As can be seen in figure 2 , above $60 \mathrm{~K}$, the heat capacity of zinc tungstate is greater than that of nickel tungstate. A mass adjustment does little to this difference.

Similar behavior has been seen previously in the study of the metal difluorides by Stout and Catalano. ${ }^{(47)}$ When the heat capacity of $\mathrm{ZnF}_{2}$ is simply subtracted from that of $\mathrm{NiF}_{2}$, the remaining heat capacity is significantly negative for several hundred kelvins. Stout and Catalano ${ }^{(47)}$ assumed the vibrational contributions to the entropy and heat capacity of the difluorides obey a law of corresponding states, scaling as the parameter $\left(T / T_{\mathrm{m}}\right)$, where $T_{\mathrm{m}}$ is the melting temperature. Such an assumption was necessary to take proper account of the large differences between the melting temperatures of the zinc and nickel difluorides.

Since the reported melting temperatures of $\mathrm{NiWO}_{4}: 1663 \mathrm{~K}^{(1)} 1723 \mathrm{~K}$, ${ }^{(48)}$ are higher than that of $\mathrm{ZnWO}_{4}: 1474 \mathrm{~K}^{(48,49)}$ and of all other tungstates, the lattice estimate must take the melting-temperature differences into consideration. The relation chosen between the $\Theta_{D}$ of nickel and zinc tungstates was the Lindemann melting formula ${ }^{(50)}$ which indicates the melting temperature $T_{\mathrm{m}}$ to be proportional 
to such structural factors as ionic masses, sizes of unit cell, and $\Theta_{D}$ each to appropriate powers:

$$
T_{m} \propto \Theta_{D}^{2} V^{2 / 3} M,
$$

The low-temperature limit of the ratio of the $\Theta_{D}$ 's of nickel and zinc tungstate was then taken as

$$
\begin{aligned}
\Theta_{\mathrm{D}}\left(\mathrm{NiWO}_{4}\right) / \Theta_{\mathrm{D}}\left(\mathrm{ZnWO}_{4}\right) & =\left[T_{\mathrm{m}}(\mathrm{Ni})\{V(\mathrm{Zn})\}^{2 / 3} M(\mathrm{Zn}) / T_{\mathrm{m}}(\mathrm{Zn})\{V(\mathrm{Ni})\}^{2 / 3} M(\mathrm{Ni})\right]^{1 / 2} \\
& =1.133 .
\end{aligned}
$$

The lowest limit was then joined to the experimental ratio of the ratio $\Theta_{\mathrm{D}}\left(\mathrm{NiWO}_{4}\right) / \Theta_{\mathrm{D}}\left(\mathrm{ZnWO}_{4}\right)$ at $150 \mathrm{~K}$. The estimated $\Theta_{\mathrm{D}}\left(\mathrm{NiWO}_{4}\right)$ lattice heat capacities were then calculated from the Debye equation and the estimated Debye $\Theta_{\mathrm{D}}$ 's.

\section{EXCESS HEAT CAPACITIES OF $\mathrm{NiWO}_{4}$ AND CoWO}

The heat capacities of these tungstates and the estimated lattice heat capacities in the vicinity of the transition temperatures are shown in figure 4 together with the



FIGURE 4. (a), (b). Heat capacities in the transition region-(a) for cobalt tungstate and (b) for nickel tungstate. $\mathrm{O}$, experimental values for $\mathrm{NiWO}_{4}$ and $\mathrm{CoWO}_{4} ; \Delta$, those for $\mathrm{ZnWO}_{4}$; the dashed curve is the adopted lattice contribution.

zinc tungstate values used for comparison with the lattice contribution. To determine the excess entropies and enthalpies, the differences between the pairs of curves were integrated. The resulting entropy and enthalpy of transition for nickel tungstate are

$$
\Delta S_{\mathrm{mag}}=2.06 \mathrm{cal}_{\mathrm{th}} \mathrm{K}^{-1} \mathrm{~mol}^{-1}, \quad \Delta H_{\mathrm{mag}}=100.2 \mathrm{cal}_{\mathrm{th}} \mathrm{mol}^{-1} .
$$

A graph of magnetic entropy for nickel tungstate is shown in figure 5(a). The entropy obtained corresponds to 94 per cent of $R \ln 3$.

The existence of a discrepancy between the expected and experimental entropies cannot be considered surprising, in view of the crudeness of the lattice approximation. Even assuming the Lindemann melting relation to be valid, the $\Theta_{D}$ ratio curve chosen 
was selected as reasonable, but is certainly not definitive. One indication of probable error in the assignment of the lattice is the fraction of entropy appearing above the Néel temperature. In three-dimensional magnetic systems, this entropy is often greater than 30 per cent of the total and continues to appear at $3 T_{\mathrm{N}}$. For the nickel

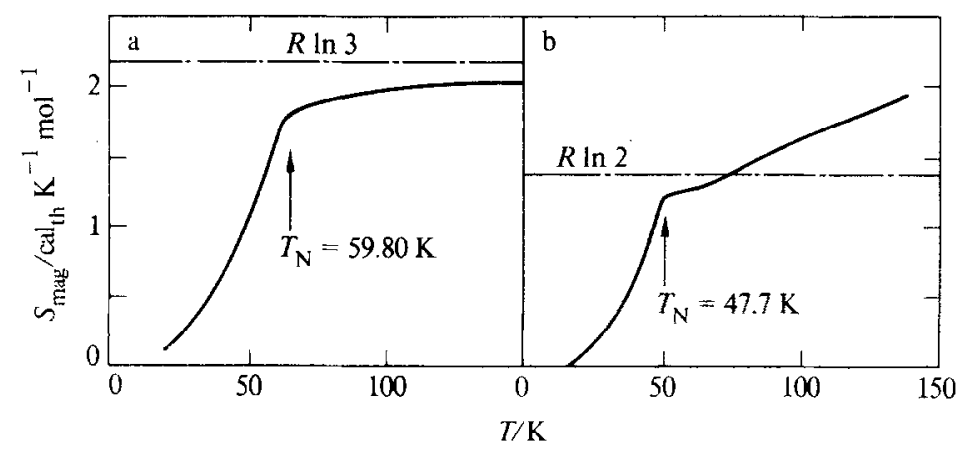

FIGURE 5. (a), (b) Magnetic entropies of (a) nickel tungstate and (b) cobalt tungstate.

tungstate only 18 per cent appears above $T_{\mathrm{N}}$. However, within the uncertainties present, the total entropy is considered as evidence for the expected spin-disorder transition in nickel tungstate.

The excess entropy and enthalpy of cobalt tungstate were calculated from the difference of the total and estimated lattice curves shown in figure 4(b) on which the heat capacity of zinc tungstate is also shown. The excess entropy is displayed in figure 5(b). The entropy approaches $R \ln 2$; then the Schottky contribution to the heat capacity causes the entropy to rise again. Nevertheless, the magnetic entropy associated with the antiferromagnetic transition is clearly $R \ln 2$, not the $R \ln 4$ predicted from the spin-only model. The entropy above $T_{\mathrm{N}}$, associated with magnetic short-range order, cannot be directly evaluated but the difference between $R \ln 2$ and the entropy which has appeared by $T_{\mathrm{N}}$ is 23 per cent of $R \ln 2$.

The excess heat capacities of cobalt and nickel tungstates show antiferromagnetic spin-wave contributions at low temperatures, as can be seen in figure 6 . From considerations of mass and volume, the lattice heat capacities of nickel and cobalt tungstate should be lower than the heat capacity of zinc tungstate. The fact that the heat capacities are larger, while still proportional to $T^{3}$, demonstrates the existence of spin-wave heat capacities for these compounds. Interestingly enough, nickel tungstate has the same initial slope as cobalt tungstate even though its transition temperature is $13 \mathrm{~K}$ higher.

Although iron(II), cobalt, and nickel tungstates consist of ferromagnetic layers aligned antiferromagnetically, there is no evidence for two-dimensional magnetic behavior in the tungstates. In $\mathrm{K}_{2} \mathrm{NiF}_{4},{ }^{(51)} \mathrm{K}_{2} \mathrm{MnF}_{4},{ }^{(52)}$ and $\mathrm{MnTiO}_{3},{ }^{(53,54)}$ anomalies in the susceptibilities have been interpreted as indicative of two-dimensional ordering, e.g. more than 45 per cent of the magnetic entropy of $\mathrm{MnTiO}_{3}$ appears above the Néel temperature. However, these three compounds all display antiferromagnetic 


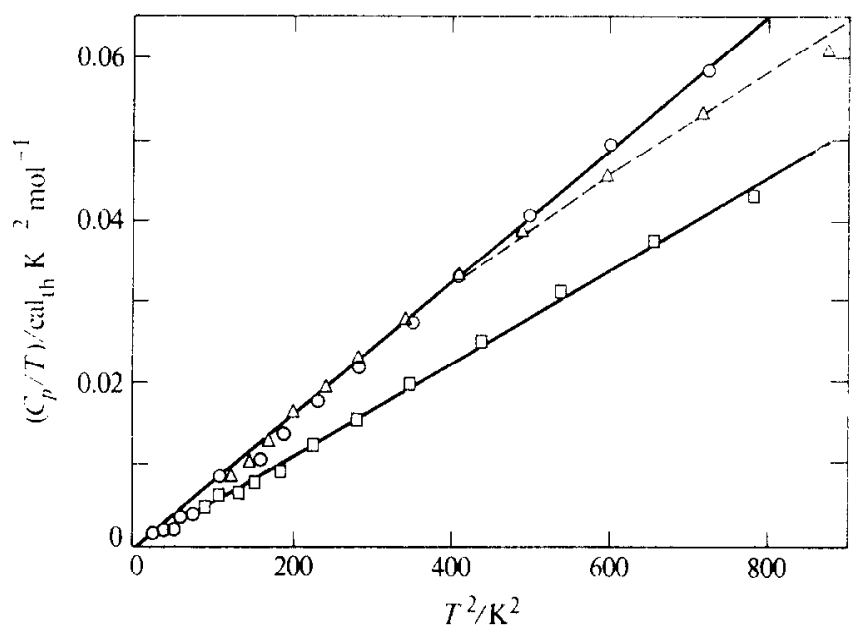

FIGURE 6. Plot of $C_{p} / T$ against $T^{2}$ for: $O$, cobalt tungstate; $\square$, nickel tungstate; $\triangle$, zinc tungstate.

coupling within the layers, unlike the $\mathrm{FeWO}_{4}, \mathrm{CoWO}_{4}$, and $\mathrm{NiWO}_{4}$. The absence of such characteristic behavior in the susceptibilities of any of the tungstates and the relatively low percentages of short-range entropy herald essentially three-dimensional exchange properties.

\section{REFERENCES}

1. Keeling, R. O. Acta. Cryst. 1957, 10, 209.

2. Sleight, A. W. Acta. Cryst. Section B, 1972, 28, 2899.

3. Van Uitert, L. G.; Sherwood, R. C.; Williams, H. J.; Rubin, J. J.; Bonner, W. A. J. Phys. Chem. Solids 1964, 25, 1447.

4. Shapovalova, R. D.; Belova, V. I.; Zalesskii, A. D.; Gerassimov, Ya. I. Russ. J. Phys. Chem. $1961,35,1340$.

5. Weitzel, H. Neus Jahrb. Mineral. Abhand. 1970, 113, 13.

6. Dachs, H.; Weitzel, H.; Stull, E. Solid State Commun. 1966, 4, 473.

7. Ulkü, D. Zeit. Fur Krist. 1967, 124, 192.

8. Weitzel, H. Solid State Commun. 1970, 8, 2071.

9. Zvyagin, A. I.: Khats'ko, E. N. Soviet Physics-Solid State 1970, 12, 258.

10. Gredescul, V. M.; Greduscul, S. A ; Eremenko, V. V.; Naukemko, V. M. J. Phys. Chem. Solids 1972, 33, 859 .

11. Eremenko, V. V. Personal communication (1975).

12. Dachs, H. Solid State Commun. 1969, 7, 1015.

13. Lyon, W. G.; Westrum, E. F., Jr. J. Chem. Thermodynamics 1974, 6, 781.

14. Landee, C. P.; Westrum, E. F., Jr. J. Chem. Thermodynamics 1975, 7, 973.

15. Broch, E. K. Norske Videnskaps-Acad. Skriften I. No. 8, 1929, 61 pp.

16. Swansen, H. E.; Morris, M. C.; Stinchfield, R. P.; Evans, E. E. Natl. Bur. Stds. (U.S.), Monograph 26, Sec. 6.2, 1963.

17. Landee, C. P., Ph-D. Thesis, The University of Michigan, Ann Arbor, Michigan 1975. Diss. Abs. $1975,36,75-29272$. Detailed supplementary information on X-ray structural patterns for some samples and detailed thermodynamic functions for all samples are presented in an NAPS document No. 02765 for 22 pages of supplementary material. Order from ASIS/NAPS c/o Microfiche Publications, 440 Park Avenue South, New York, N.Y. 10016 U.S.A. Remit in advance for each NAPS accession number. Make checks payable to Microfiche Publications. Photocopies are \$5.50. Microfiche are \$3.00. Outside of the U.S. and Canada, postage is $\$ 2.00$ for a photocopy or $\$ 1.00$ for a fiche. 
18. Powder Data File, Joint Committee on Powder Diffraction Standards, 1845 Walnut Street, Philadelphia, Pennsylvania 19103, U.S.A. Card No. 4-0835.

19. Reference 18. Card No. 7-210.

20. Reference 18. Card No. 92-772.

21. Westrum, E. F., Jr.; Furukawa, G. T.; McCullough, J. P. Adiabatic low-temperature calorimetry in Experimental Thermodynamics, Vol. I. McCullough, J. P.; Scott, D. W., editors. Butterworths: London, 1968.

22. West, E. D.; Westrum, E. F., Jr. Adiabatic calorimetry from 300 to $800 \mathrm{~K}^{\prime \prime}$. in Experimental Thermodynamics, Vol. I. McCullough, J. P.; Scott, D. W., editors. Butterworths: London, 1968.

23. Westrum, E. F., Jr., Unpublished results.

24. Lyon, W. G.; Westrum, E. F., Jr. J. Chem. Thermodynamics 1968, 49, 3374.

25. King, E. G.; Weller, W. W. U.S. Bur. Mines Rept. Invest., No. 5791, 1961.

26. Anderson, P. W. Theory of magnetic exchange interactions: exchange in insulators and semiconductors. In Solid State Physics. Seitz, F.; Turnbull, D., editors. Academic Press: New York. 1963.

27. Anderson, P. W. Exchange in insulators: superexchange, direct exchange, and double exchange. In Magnetism, Vol. 1. Rado, G. T.; Suhl, H., editors. Academic Press: New York. 1963.

28. Grime, H.; Santos, J. A. Z. Kristallogs. Mineral. 1934, 88, 136.

29. Goodenough, J. B. Magnetism and the Chemical Bond. Interscience: New York. 1963.

30. Wilkinson, M. K.; Cable, J. W.; Wollan, E. O.; Koehler, W. C. Phys. Rev. 1959, 113, 497.

31. Wilkinson, M. K.; Cable, J. W.; Wollan, E. O.; Koehler, W. C. Oak Ridge National Laboratory Report ORNL-2501, 1958 (unpublished); Oak Ridge National Laboratory Report ORNL-2430. 1959 (unpublished).

32. Cid-Dresdner, H.; Escobar, C. Z. Kristallogr. Mineral 1968, 127, 61.

33. Lesne, J. P.; Caillet, P. Can. J. Spect. 1973, 18, 69.

34. Blasse, G. Proceedings of the International Conference of Magnetism. Institute of Physics and the Physical Society: London. 1964, p. 350.

35. Ferguson, J.; Wood, D. L.; Knox, K. J. Chem. Phys. 1963, 39, 881.

36. Ferguson, J.; Knox, K.; Wood, D. L. J. Chem. Phys. 1961, 35, 2236.

37. Lyon, W. G., Ph.D. Thesis, The University of Michigan, Ann Arbor, Michigan 1973. Diss. Abs. 1974, 35, 74-15790.

38. Shannon, R. D.; Vincent, H. Interatomic Distances and Magnetic properties in halides and chalcogenides. In Structure and Bonding 19. Dunitz, J. D.; Hemmerich, P.; Holm, R. H.; Ibers, J. A.; Jorgensen, C. K.; Neilands, J. B.; Reinen, D.; Williams, R. J. P., editors. SpringerVerlag: New York. 1974.

39. Reynolds, M. L.; Hayston, W. F.; Garlick, G. F. Phys. Status Solidi 1968, 20. 735.

40. Atsarkin, V. A.; Morshnev, S. K.; Potkin, L. I. Soviet Physics-Solid State 1967, 9, 660.

41. Zvyagin, A. 1.; Khats'ko, E. N. Soviet Plastics-Solid State 1969, 10, 2970.

42. Bates, C. A.; Oglesby, M. J.; Standley, K. J. J. Phys. C. 1972, 5, 2949.

43. Galkin, A. A.; Prokhorov, A. D.; Tsintsudze, G. A. Soviet Plastics-Solid State 1970, 12, 1414.

44. See for example Lewis, G. N.; Randall, M. Thermodynamics revised by Pitzer, K. S.; Brewer, L., editors, second edition. McGraw-Hill Book Co., Inc. : New York. 1961, chapter 27.

45. Stout, J. W. Pure Appl. Chem. 1961, 2, 287.

46. Mannheim, P. D.; Simopoulos, A. Phys. Rev. 1968, 165, 845; and Nussbaum, R. H.; Howard, D. G.; Nees, W. L.; Steen, C. F. Phys, Rev. 1968, 173, 653.

47. Stout, J. W.; Catalano, E. J. Chem. Phys. 1955, 23, 2013.

48. Schroecke, H. Neus. Jahrb. Mineral., Abhand. 1969, 110, 115.

49. O'Hara, S.; McManus, G. M. J. Appl. Phys. 1965, 36, 1741.

50. Ziman, J. M. Principles of the Theory of Solids. Cambridge University Press: Cambridge, England. 1969, p. 63.

51. Srivastava, K. G. Phys. Lett 1963, 4, 55.

52. Breed, D. J. Phys. Lett. 1966, 23, 181.

53. Akimitsu, J.; Ishikawa, Y.; Endoh, Y. Solid State Commun. 1970, 8, 8187.

54. Goodenough, J. B.; Stickler, J. J. Phys. Rev. 1967, 164, 768. 